\title{
Chronic melatonin administration reduced oxidative damage and cellular senescence in the hippocampus of a mouse model of Down syndrome
}

Eduardo B. Parisottoa, Verónica Vidalb, Susana García-Cerrob, Sara Lantiguab, Danilo W. Filhoa,

Emilio J. Sanchez-Barcelób, Carmen Martínez-Cuéb, Noemí Ruedab*.

a Department of Ecology and Zoology, Federal University of Santa Catarina, Florianópolis, Brasil

b Department of Physiology and Pharmacology, School of Medicine, University of Cantabria,

39011 Santander, Spain

${ }^{*}$ Corresponding author:

Noemí Rueda

Laboratory of Neurobiology of Learning

Department of Physiology and Pharmacology

Faculty of Medicine

University of Cantabria

c/ Cardenal Herrera Oria, s/n

Santander, 39011

SPAIN

Email: ruedan@unican.es

Tel: +34-942201966

Fax: +34-942201903 


\section{Abstract}

Previous studies have demonstrated that melatonin administration improves spatial learning and memory and hippocampal long-term potentiation in the adult Ts65Dn (TS) mouse, a model of Down syndrome (DS). This functional benefit of melatonin was accompanied by protection from cholinergic neurodegeneration and the attenuation of several hippocampal neuromorphological alterations in TS mice. Because oxidative stress contributes to the progression of cognitive deficits and neurodegeneration in DS, this study evaluates the antioxidant effects of melatonin in the brains of TS mice. Melatonin was administered to TS and control mice from 6 to 12 months of age and its effects on the oxidative state and levels of cellular senescence were evaluated. Melatonin treatment induced antioxidant and antiaging effects in the hippocampus of adult TS mice. Although melatonin administration did not regulate the activities of the main antioxidant enzymes (superoxide dismutase, catalase, glutathione peroxidase, glutathione reductase, and glutathioneS-transferase) in the cortex or hippocampus, melatonin decreased protein and lipid oxidative damage by reducing the thiobarbituric acid reactive substances (TBARS) and protein carbonyls (PC) levels in the TS hippocampus due to its ability to act as a free radical scavenger. Consistent with this reduction in oxidative stress, melatonin also decreased hippocampal senescence in TS animals by normalizing the density of senescence-associated $\beta$-galactosidase positive cells in the hippocampus. These results showed that this treatment attenuated the oxidative damage and cellular senescence in the brain of TS mice and support the use of melatonin as a potential therapeutic agent for age-related cognitive deficits and neurodegeneration in adults with DS.

Keywords: Down syndrome; melatonin; Ts65Dn mice; cellular senescence; oxidative stress. 


\section{Introduction}

Down syndrome (DS) is characterized by a triplication of a complete or partial copy of chromosome 21 (Hsa21). The cognitive impairment of DS individuals is partially due to developmental alterations, although in later life stages, this impairment is progressively aggravated due to accelerated aging and the development of Alzheimer's disease (AD) neuropathology $[1,2]$.

Among the different mouse models of DS, the Ts65Dn (TS) mouse, that contains three copies of 92 genes orthologous to Hsa21 genes [3], recapitulate numerous phenotypic characteristics of DS, including cognitive deficits and alterations in brain morphology and function [1, 4], that become more pronounced as the animals age [5].

One of the mechanisms that contributes to the accelerated aging, cognitive and neuronal dysfunction in DS is increased oxidative stress that is present from early life stages affecting neurogenesis and differentiation, connection and survival [6-8]. During later life stages, oxidative stress is aggravated in DS and in the TS mouse [9-12] contributing to their progression of cognitive and neuronal degeneration $[4,9,13]$.

The enhanced oxidative stress found in DS individuals and in the TS mouse is caused by the triplication of several Hsa21 genes [9] including the SOD1/Sod1, the gene responsible for the formation of superoxide dismutase (SOD), which catalyzes the conversion of superoxide anions into hydrogen peroxide $\left(\mathrm{H}_{2} \mathrm{O}_{2}\right)$. The increase in $\mathrm{SOD}$ activity results in the formation of disproportionate levels of $\mathrm{H}_{2} \mathrm{O}_{2}$, leading to the overproduction of highly reactive oxygen species (ROS). In addition, oxidative stress induces cell senescence [14, 15], a process that is characterized by permanent arrest of cell proliferation [16].

Melatonin is an indoleamine mainly synthesized and secreted by the pineal gland. Its production progressively decreases as animals age [17], and its exogenous administration has been demonstrated to induce neuroprotective effects [18-20]. Melatonin protects against oxidative 
stress regulating anti- and pro-oxidant enzymes, acting as a potent ROS scavenger [21] and repairing molecules damaged by ROS overgeneration. Thus, melatonin has been proposed to be a powerful tool in the treatment of neuropathologies in which oxidative stress is enhanced.

Our previous studies [10, 22], showed that melatonin treatment during adulthood improved spatial memory and hippocampal long-term potentiation (LTP) in TS mice. This functional benefit was associated with protection against cholinergic neurodegeneration and the normalization or attenuation of several hippocampal neuromorphological alterations of TS mice. The aim of this study was to evaluate the oxidative status and the density of senescent cells in the brain of adult TS mice and to evaluate the effect of chronic melatonin treatment on these processes. 


\section{Materials and methods}

\section{Animals and housing}

This study was approved by the Cantabria University Institutional Laboratory Animal Care and Use Committee and was carried out in accordance with the Declaration of Helsinki and the European Communities Council Directive (86/609/EEC). Mice were generated, karyotyped, housed and maintained as previously described Corrales et al. [22].

\section{Melatonin treatment and experimental groups}

TS and euploid littermates (CO) mice were orally treated with melatonin (Mel: $100 \mathrm{mg} / \mathrm{L}$; SigmaAldrich, Madrid, Spain) or its diluent from 6 to 12 months of age as previously described Corrales et al. [10] and assigned to one of the following experimental groups: TS-Mel, CO-Mel, TS-vehicle or CO-vehicle. Six animals per group were used to assess the effects of melatonin administration on the oxidative stress assessments, while 7 extra animals per group were used to perform the senescence study.

\section{Oxidative stress assays}

Sample preparation. Cortex and hippocampal samples were homogenized in cold buffer containing $20 \mathrm{mM}$ sodium phosphate, $\mathrm{pH}$ 7.4; $0.1 \%$ Triton; and $150 \mathrm{mM} \mathrm{NaCl}(1: 20 \mathrm{w} / \mathrm{v})$ and centrifuged at $5000 \mathrm{~g}$ for $5 \mathrm{~min}$. The supernatant was used to perform all the biochemical determinations that were carried out in triplicates.

Antioxidant enzyme assays. Enzymatic activities were measured as described by Parisotto et al. [23]. Briefly, CAT activity was analyzed at $240 \mathrm{~nm}$ quantifying the decrease in the level of $\mathrm{H}_{2} \mathrm{O}_{2}$ (expressed in $\mathrm{mmol} / \mathrm{min} / \mathrm{g}$ ) in a $10 \mathrm{mM} \mathrm{H}_{2} \mathrm{O}_{2}$ solution. In order to determine SOD activity, the oxidation of epinephrine ( $\mathrm{pH} 2.0$ to $\mathrm{pH} 10.2)$, which produces superoxide anion and a pink chromophore (expressed in USOD/g), was quantified at $480 \mathrm{~nm}$. GPx activity was determined 


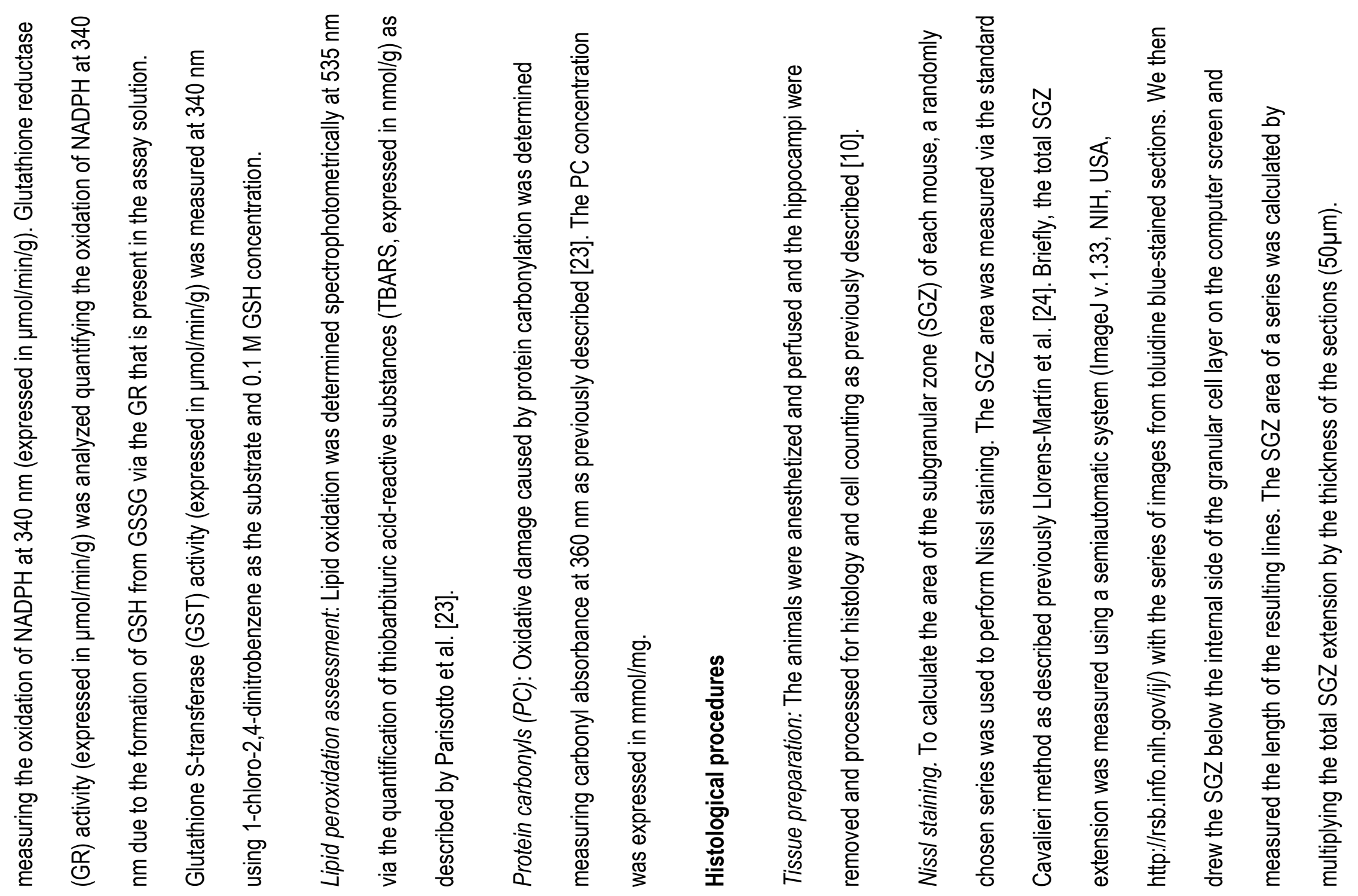

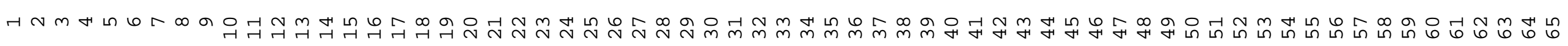


Histochemical detection of senescence-associated $\beta$-galactosidase. To estimate the density of senescent cells in the SGZ of the dentate gyrus (DG) in the different groups of mice we used the SA- $\beta$-gal assay (senescence-associated $\beta$-galactosidase) method described by $\mathrm{He}$ et al. [25]. The hippocampal sections were washed twice with PBS and fixed for $15 \mathrm{~min}$ at room temperature with a $0.5 \%$ glutaraldehyde solution. Next, the sections were washed and incubated with a staining solution containing 5-bromo-4chloro-3-indolyl-ß-D-galactopyranoside (X-gal, Thermofisher Scientific, MA, USA) for $24 \mathrm{~h}$ at $37^{\circ} \mathrm{C}$, mounted on Superfrost plus glass slides, dehydrated, cleared, and coverslipped with mounting medium. The density of SA- $\beta$-gal- positive cells (showing a blue reaction product over the cell soma) was determined by counting all blue cells in the SGZ of the DG of each animal using a Zeiss Axioskop 2 plus microscope with a 40X objective and dividing this number by the SGZ area.

\section{Statistical analysis}

Data were analyzed using MANOVA ('genotype' x 'treatment') followed by post-hoc group comparisons after Bonferroni corrections when all groups were compared and Student's t-test when two individual groups were compared. All of the analyses were performed in SPSS (version 22.0, Chicago, IL, USA) for Windows. 


\section{Results}

To determine the brain oxidative stress status of adult TS mice and the possible beneficial effects of chronic administration of melatonin, we evaluated the levels of brain oxidative damage and the state of the antioxidant enzymatic system in the hippocampus and cortex of vehicle- and melatonin-treated TS and CO mice. Table 1 shows the results of the multivariance analysis on each variable assessed.

Table 1. $F$ values of MANOVA (genotype $x$ treatment) for oxidative stress markers and antioxidant enzymatic activities in the cortex and hippocampus of the four groups of animals.

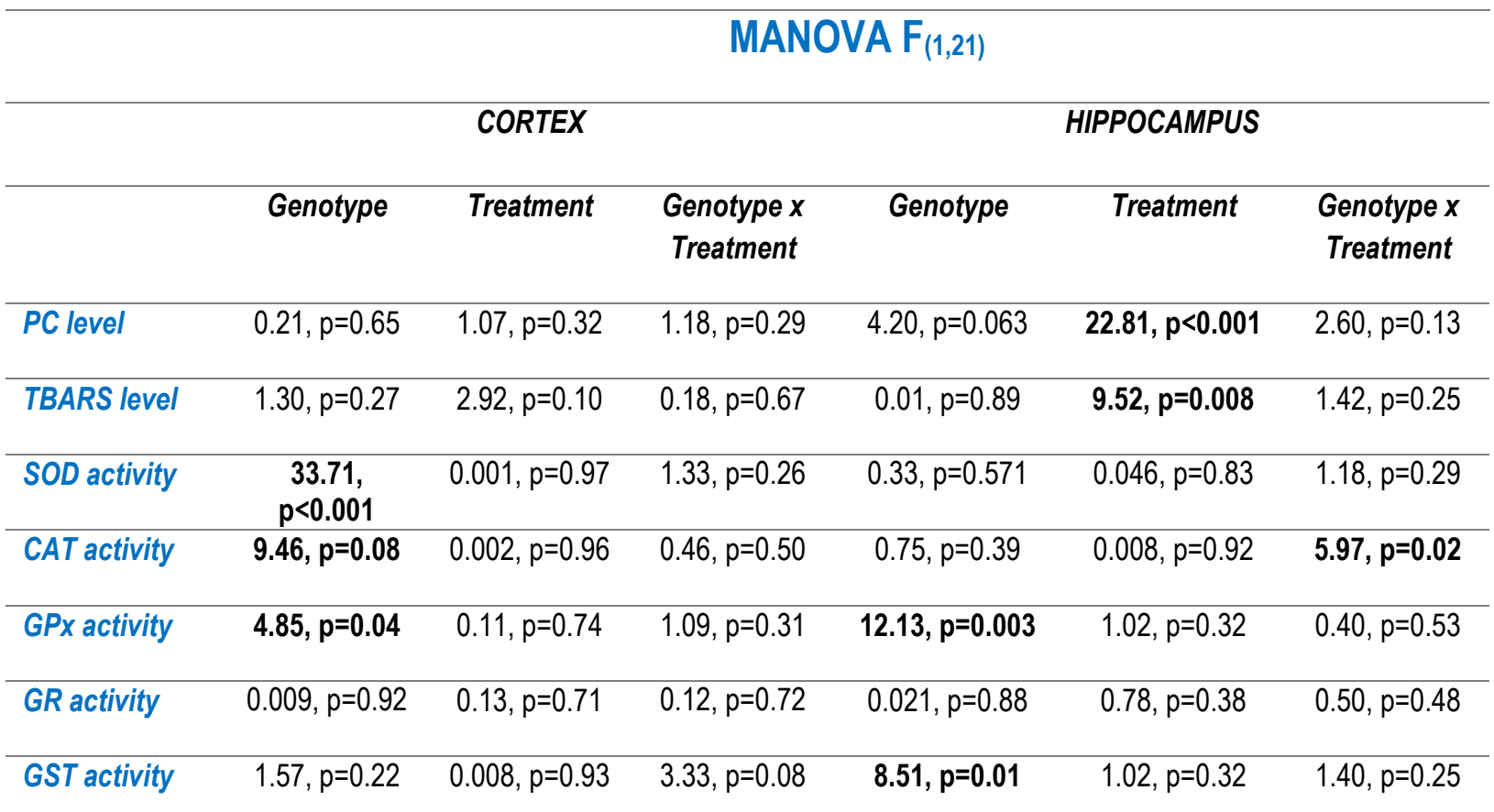

To evaluated the effects of melatonin treatment on the levels of brain oxidative damage, we measured the levels of PC (a marker of protein damage induced by ROS) and of TBARS (a marker of lipid damage induced by ROS or lipid peroxidation).

Although MANOVA revealed no significant differences due to the genotype, post hoc analyses showed a significant increase in the level of oxidized proteins (PC, $\sim 10 \% ; t=2.48 ; p=0.038$; Fig. $1 \mathrm{~A}$ ) and of lipid peroxidative damage (TBARS, $16 \% ; t=2.33 ; p=0.042 ;$ Fig. $1 \mathrm{~B}$ ) in the 
hippocampus of adult vehicle-treated TS mice compared to CO mice under the same treatment.

However, no differences in the cortical levels of both markers were observed between the different groups of animals (Fig. 1A and B). Melatonin significantly decreased the levels of protein damage $(\sim 12 \% ; t=5.86 ; p=0.001)$ and lipid peroxidation $(\sim 29 \% ; t=5.14 ; p<0.001)$ in the hippocampus of TS mice (Fig. 1A and B, Table 1) and produced a significant decrease in the levels of PC in the hippocampus of CO mice ( 10\%; $t=2.68 ; p=0.028$; Fig 1A, Table 1).

Next, we examined whether melatonin might also exert its antioxidant effects by regulating the activity of the most important antioxidant enzymes in the cortex and hippocampus of the animals. As expected, SOD activity was increased $(\sim 143 \%)$ in the cortex of TS mice treated with vehicle compared to their CO littermates (Fig. 2A, Table 1). In the hippocampus, although MANOVA revealed the absence of significant differences due to genotype, post hoc comparisons showed higher SOD activity in vehicle-treated TS mice than in CO mice ( 33\%) under the same treatment $(t=2.31 ; p=0.046)$. Melatonin administration did not modify SOD activity in either brain region (Table 1).

We then analyzed the activity of CAT and GPx because the activity of SOD must be coordinated with the activity of these two antioxidant enzymes to metabolize the $\mathrm{H}_{2} \mathrm{O}_{2}$ that is produced by SOD into $\mathrm{H}_{2} \mathrm{O}$ and $\mathrm{O}_{2}$. As shown in Fig. $2 \mathrm{~B}$, CAT activity was increased in the cortex ( $\left.64 \%\right)$ but not in the hippocampus of TS animals. In addition, GPx activity was increased in both brain structures (cortex: 34\%; hippocampus 27\%, Fig. 2C) in vehicle-treated TS mice when compared to $\mathrm{CO}$ animals (Table 1). Chronic melatonin administration did not modify the activity of CAT or GPx in the cortex or hippocampus of TS or CO mice (Figs. 2B and C, Table 1).

To detoxify hydroperoxides, GPx requires the participation of glutathione (GSH) as a co-factor to counteract the continuous formation of its oxidized form (GSSG), which is very toxic to cells. Thus, we also measured the activity of GR, a flavoprotein that allows the conversion of GSSG 
back into GSH. GR activity did not differ in the cortex or hippocampus of TS or CO mice that were treated with melatonin or vehicle during adulthood (Fig. 2D, Table 1).

Finally, we measured the activity of GST, an enzyme that participates in the detoxification of the endogenous hydroperoxides continuously generated through cellular lipoperoxidation processes. TS mice presented less hippocampal GST activity $(\sim 12 \%)$ than CO mice that received the same treatment. However, GST activity was similar in the cortex of the different groups of mice (Fig. 2E, Table 1).

Because oxidative stress induces premature cellular senescence, we assessed the density of SA$\beta$-gal-positive cells in the hippocampus of the four groups of mice. TS mice treated with vehicle presented a higher density of $\beta$-gal-positive cells than $\mathrm{CO}$ mice, indicating greater hippocampal senescence (MANOVA 'genotype': $F_{(1,25)}=6.35, p=0.019$; Fig. 3). Interestingly, melatonin treatment significantly reduced the density of cells with a senescent phenotype in the DG of TS mice (MANOVA 'treatment': $F_{(1,25)}=9.07, p=0.005$; Fig. 3B). 


\section{Discussion}

We have previously demonstrated that melatonin exerts cognitive-enhancing effects in the adult TS mouse [10,22]. These beneficial effects of melatonin in TS animals were partially due to the prevention of cholinergic degeneration and to the normalization of the function and/or morphology of the hippocampus. In this study, we evaluated other mechanisms that could be involved in the altered cognition of these mice, i.e., the oxidative stress status and the density of senescent cells in the brain, as well as the effect of melatonin treatment on these processes.

We first evaluated the status of the antioxidant defense system in the brain of TS and CO mice. Consistent with the triplication of the Sod 1 gene and the increased activity of SOD observed in different tissues in DS individuals and TS mice [15, 23, 26, 27], we found that SOD activity was increased in the cortex and hippocampus of TS mice. Although SOD catalyzes the dismutation of superoxide, a free radical with high toxicity, to non-radical molecules such as oxygen and $\mathrm{H}_{2} \mathrm{O}_{2}$, the accumulation of $\mathrm{H}_{2} \mathrm{O}_{2}$ or its inefficient removal, leads to the formation of the most deleterious hydroxyl radical (HO-) that damage membrane lipids, proteins and other biomolecules [28]. Thus, the increase activity of SOD found in the brain of TS mice may lead to an excess of $\mathrm{OH}$ production resulting in high oxidative damage.

To prevent the accumulation of $\mathrm{H}_{2} \mathrm{O}_{2}$, SOD activity must be balanced with GPx and CAT activities. In DS neurons, higher SOD activity without the concomitant increase of complementary antioxidant enzymes activities, CAT and GPx, create a redox imbalance that may not efficiently neutralize the excess of $\mathrm{H}_{2} \mathrm{O}_{2}$ [29]. In this study, while GPx activity was increased in the cortex and hippocampus, CAT activity was only increased in the cortex of TS mice. The increase in GPX activity found in the brains of TS mice may be due to induction of the enzyme by excess $\mathrm{H}_{2} \mathrm{O}_{2}$ and lipid peroxides as an adaptative response to oxidative stress [30]. However, the fact that CAT activity was not increased in the hippocampus of TS mice to compensate the enhanced SOD 
activity could result in insufficient removal of $\mathrm{H}_{2} \mathrm{O}_{2}$, which would favor the generation of $\mathrm{HO}$; this, in turn, could produce persistent oxidative stress.

Among the anti-oxidative stress-related enzymes, GST acts in xenobiotic detoxification by catalyzing the conjugation of GSH to chemical toxins [31] and contributing to the detoxification of the endogenous hydroperoxides that are continuously generated through cellular lipoperoxidation processes [32]. In agreement with previous observations in the blood of DS subjects [27, 31], in this study, the activity of the GST enzyme in the hippocampus of TS mice was lower than that in CO mice. Considering the antioxidant effects of this enzyme [31], this may be an additional factor contributing to the oxidative damage in this structure in this model of DS.

The levels of GSH, another major neuronal endogenous antioxidant, are reduced in DS subjects $[27,31]$. Consistent with previous studies [33], we found no differences in the brain activity of GR (a central player in the conversion of GSSH to GSH) between CO and TS mice. However, the levels of GSH also depend on the levels of y-glutamylcysteine synthase and glucose-6-phosphate dehydrogenase [34] that were not measured in our study.

Melatonin has been shown to reduce oxidative stress through different mechanisms [21, 34, 35]: indirectly, by modulating the activity of the enzymes that are involved in controlling oxidative processes (up-regulating antioxidant and down-regulating pro-oxidant enzymes); and directly, by acting as a free radical scavenger and interacting with oxidative radicals.

In this study, chronic melatonin treatment did not modify the activity of SOD, CAT, GPx, GR or GST enzymes, suggesting that melatonin does not exert its antioxidant effects in the brains of adult TS mice by regulating the antioxidant defense system. This lack of effect of melatonin to modulate the indirect mechanism to reduce oxidative stress in TS mice might be due to the fact that the ability of this indoleamine to detoxify free radicals is highly variable and depends on the tissues and species involved [35, 36]. For example, Olcese et al. [37] found that long-term 
melatonin administration to a mouse model of $A D$ diminish the mRNA expression levels of the antioxidant enzymes SOD, CAT and GPx. However, a previous study in another model of AD reported opposite effects of melatonin treatment on antioxidant enzymes [38]. In TS mice, the genetically predetermined increase in SOD activity induces a dysregulation of the other two enzymes and other triplicated genes also influence the redox-metabolism [39]. These factors are likely to be contributing to the divergent results found after melatonin administration in the expression levels of these enzymes in TS mice and in murine models of $A D$. Furthermore, the regulation of antioxidant enzymes by melatonin can be also different depending upon under basal or elevated oxidative stress conditions [34] as occur in the brain of TS mice.

Melatonin can also contribute to neuroprotection exerting other indirect antioxidative and prosurvival effects by regulating different anti- or pro- apoptotic proteins and enzyme cofactors [ 40 , 41]. In addition, melatonin also modulates a broad set of ROS- and survival-related signaling pathways that implicate transcriptional factors, such as NF-kB or Akt [42- 44], that activate different pro-inflammatory genes involved in age-related processes under increased oxidative stress. Future studies should explore the effects of melatonin administration in these signaling pathways in TS mice.

Lipids and proteins are the molecules that are most prone to undergo major oxidative injury. Therefore, we measured the levels of oxidative damage in proteins and lipids in the brains of TS mice. Consistent with previous studies in DS individuals and TS mice in which other markers of oxidative stress were analyzed [10,12], the levels of TBARS and PC were increased in the hippocampus, but not in the cortex, of vehicle-treated TS mice. These findings suggest that in adult TS mice, cells in the cortex may exhibit greater tolerance or better regulation against oxidative stress than in the hippocampus. Further support for this hypothesis comes from the finding that the activity of CAT and GPx was also increased in the cortex of TS mice. These effects may compensate for the enhanced SOD1 activity, leading to a reduction in the production 
of ROS and of oxidative injury in the cortex. Conversely, an imbalance of these enzymes in the hippocampus would lead to increased oxidative stress in this structure, which could play a role in the cognitive deficits and neurogenesis alterations that have previously been described in this model of DS [4]. Therefore, further studies are needed to determine whether brain structurespecific manipulations of the levels of the SOD and catalase enzymes, may account for the higher vulnerability of hippocampus to suffer oxidative stress damage.

The second mechanism by which melatonin reduces oxidative damage is its direct ROS scavenging properties [21], detoxifying a variety of free radicals and reactive oxygen intermediates, including $\mathrm{OH}$, peroxynitrite anion, singlet oxygen and nitric oxide [45] to avoid oxidative damage. In this study we showed that melatonin treatment rescued the levels of protein damage and lipid peroxidation in the hippocampus of TS mice because they did not differ from those of $\mathrm{CO}$ mice, indicating that the main antioxidant action of this indoleamine in this mouse model of DS may be due to its action as a free radical scavenger. This protective effect of melatonin against lipid and protein damage is consistent with previous reports in other mouse models with different neuropathologies $[20,38]$ or with brain damage induced by radiation or by toxin exposure $[46,47]$ in which oxidative stress plays an important role.

In addition, oxidative stress is an important factor that causes cell senescence $[14,15,25]$. Because the hippocampus of TS mice seems to be exposed to greater amounts of oxidative stress, we analyzed the density of senescent cells in this structure. We found that vehicle-treated TS mice showed a higher density of SA- $\beta$-gal-positive cells in the DG of the hippocampus than CO mice. Consistent with these findings, it was recently demonstrated that fibroblasts with trisomy 21 present signs of premature cell senescence secondary to increased oxidative damage [15]. 
Interestingly, melatonin normalized the density of SA- $\beta$-gal-positive cells in TS mice to a level comparable to the one found in vehicle-treated CO mice, indicating a potential antiaging protective effect in the hippocampus of this model. These results are consistent with previous studies that showed that melatonin treatment effectively reverses $\mathrm{H}_{2} \mathrm{O}_{2}$-induced senescent phenotypes in mesenchymal stem cells [14]. Thus, it is likely that the melatonin-induced antioxidant effects in the hippocampus of TS mice may be involved in the reduction of senescent cells in this structure.

Although administration of the antioxidant analogue of the nootropic piracetam SGS-111 from conception or during adulthood failed to improve cognition in the TS mouse [48], other antioxidants, such as vitamin E, normalize oxidative stress and delay impairments in cognitive performance [11]. It has been demonstrated that, due to a variety of physiological and metabolic advantages [35], the protective effects of melatonin against oxidative damage are more potent than the ones induced by vitamins $C$ or $E$ [21], partially because the metabolites formed by free radical scavenging also present antioxidant activity [21, 35]. Because the pro-cognitive effects of melatonin are related to its multiple antioxidant actions $[20,37,46,47]$ and the learning and memory deficits in TS mice are associated with increased brain oxidative stress, our results suggests that the beneficial effects found in TS mice cognition after melatonin treatment [22] could be partially due to its antioxidant action as a ROS scavenger.

However, because melatonin has been also demonstrated to exert other neuroprotective effects, its administration could improve cognition by other mechanisms such as recovering LTP or promoting neurogenesis [19, 20, 49-51]. In our previous work [10], melatonin also decreased cholinergic degeneration, increased the density of proliferating cells, of differentiated neuroblast and of mature granular cells and improved the impaired LTP in the hippocampus of the TS mice. And, in the present study, melatonin also reduced the density of cells with a senescent phenotype in TS mice. These results support the idea that the pro-cognitive effects of this indoleamine could 
also be due to other neuroprotective actions. Therefore, melatonin could be a more effective pharmacological tool to reduce several DS-altered phenotypes than other antioxidants due to its multiple effects.

\section{Conclusions}

In summary (see Fig. 4), the results of the present study revealed an enhanced pro-oxidant status in the hippocampus of adult TS mice. This persistent oxidative state may account for the higher density of cells with a senescent phenotype that was demonstrated for the first time in the hippocampus of TS mice. Melatonin administration exerted antioxidant effects in the hippocampus of TS animals that were apparently not mediated by regulation of the activity of the antioxidant defense system but by the effects of melatonin as a ROS scavenger, which led to attenuation of the levels of oxidative damage. In addition, melatonin exerted an antiaging effect, as demonstrated by a reduction in the density of senescent cells in the hippocampus of TS mice. The present results provide further support for the neuroprotective effects of melatonin administration to adult TS animals and suggest that melatonin could be a potential beneficial supplement for the treatment of the age-related cognitive decline in DS individuals.

\section{Acknowledgments}

This work was supported by the Jerome Lejeune Foundation and the Spanish Ministry of Economy and Competitiveness (PSI2012-33652) and by a grant from CNPq/Brazil (proc. 2606/14-13).

\section{Author contributions}

E.B.P. and S.G. performed the experiments. V.V. and S.L. developed the colony and karyotyped the animals. D.W.F. and E.S.-B. discussed the experiments. C.M.-C. and N.R. conceived the project; designed, supervised and interpreted the experiments; analyzed the data; and wrote the manuscript. 


\section{Conflicts of interest}

The authors report no conflicts of interest. The authors alone are responsible for the content and

writing of the paper 


\section{References}

[1] Bartesaghi R, Guidi S, Ciani E. Is it possible to improve neurodevelopmental abnormalities in Down syndrome? Rev Neurosci. 22 (2011) 419-455.

[2] Lott IT, Head E, Doran E, et al. Beta-amyloid, oxidative stress and down syndrome. Curr Alzheimer Res. 3 (2006) 521-528.

[3] Sturgeon X, Gardiner KJ. Transcript catalogs of human chromosome 21 and orthologous chimpanzee and mouse regions. Mamm Genome. 22 (2011) 261-227.

[4] Rueda N, Flórez J, Martínez-Cué C. Mouse models of Down syndrome as a tool to unravel the causes of mental disabilities. Neural Plast. 2012; 2012:584071.

[5] Hamlett ED, Boger HA, Ledreux A, et al. Cognitive Impairment, Neuroimaging, and Alzheimer Neuropathology in Mouse Models of Down Syndrome. Curr Alzheimer Res. 13 (2016) 35-52.

[6] Perluigi M, du Domenico F, Fiorini A, et al. Oxidative stress occurs early in Down syndrome pregnancy: a redox proteomics analysis of amniotic fluid. Proteomics Clin Appl. 5 (2011) 167-168.

[7] Busciglio J, Yankner BA. Apoptosis and increased generation of reactive oxygen species in Down's syndrome neurons in vitro. Nature 378 (1995) 776-779.

[8] Pelsman A, Hoyo-Vadillo C, Gudasheva TA, et al. GVS-111 prevents oxidative damage and apoptosis in normal and Down's syndrome human cortical neurons. Int J Development Neurosci. 21 (2003)117124.

[9] Perluigi M, Butterfield DA. Oxidative Stress and Down Syndrome: A Route toward Alzheimer-Like Dementia. Curr Gerontol Geriatr Res. 2012; 2012:724904.

[10] Corrales A, Vidal R, García S, et al. Chronic melatonin treatment rescues electrophysiological and neuromorphological deficits in a mouse model of Down syndrome. J Pineal Res. 56 (2014) 51-61.

[11] Lockrow J, Prakasam A, Huang P, et al. Cholinergic degeneration and memory loss delayed by vitamin E in a Down syndrome mouse model. Exp Neurol. 216 (2009) 278-289.

[12] Shichiri M, Yoshida Y, Ishida N, et al. a-Tocopherol suppresses lipid peroxidation and behavioral and cognitive impairments in the Ts65Dn mouse model of Down syndrome. Free Radic Biol Med. 50 (2011) 1801-1811.

[13] Busciglio J, Pelsman A, Helguera P, et al. NAP and ADNF-9 protect normal and Down's syndrome cortical neurons from oxidative damage and apoptosis. Curr Pharm Des. 13 (2007) 1091-1098.

[14] Zhou L, Chen X, Liu T, et al. Melatonin reverses $\mathrm{H}_{2} \mathrm{O}_{2}$-induced premature senescence in mesenchymal stem cells via the SIRT1-dependent pathway. J Pineal Res. 59 (2015) 190-205.

[15] Rodríguez-Sureda V, Vilches Á, Sánchez O, et al. Intracellular oxidant activity, antioxidant enzyme defense system, and cell senescence in fibroblasts with trisomy 21. Oxid Med Cell Longev 2015; 2015:509241.

[16] Vidal MA, Walker NJ, Napoli E, et al. Evaluation of senescence in mesenchymal stem cells isolated from equine bone marrow, adipose tissue, and umbilical cord tissue. Stem Cells Dev. 21 (2012) 273-283.

[17] Míguez JM, Recio J, Sánchez-Barceló E, et al. Changes with age in daytime and nighttime contents of melatonin, indoleamines, and catecholamines in the pineal gland: a comparative study in rat and Syrian hamster. J Pineal Res. 25 (1998) 106-115.

[18] Ramirez-Rodriguez G, Ortiz-Lopez I, Dominguez-Alonso A, et al. Chronic treatment with melatonin stimulates dendrite maturation and complexity in adult hippocampal neurogenesis of mice. J Pineal Res. 50 (2011) 29-37.

[19] Liu XJ, Yuan L, Yang D, et al. Melatonin protects against amyloid- $\beta$-induced impairments of hippocampal LTP and spatial learning in rats. Synapse 67 (2013) 626-636. 
[20] Yoo DY, Kim W, Lee CH, et al. Melatonin improves D-galactose-induced aging effects on behavior, Res. 52 (2012) 21-28.

[21] Galano A, Tan DX, Reiter RJ. Melatonin as a natural ally against oxidative stress: a physicochemical examination. J Pineal Res. 51 (2011) 1-16.

[22] Corrales A, Martínez P, García S, et al. Long-term oral administration of melatonin improves spatial learning and memory and protects against cholinergic degeneration in middle-aged Ts65Dn mice, a model of Down syndrome. J Pineal Res. 54 (2013) 346-358.

[23] Parisotto EB, Giaretta AG, Zamoner A, et al. Persistence of the benefit of an antioxidant therapy in children and teenagers with Down syndrome. Res Dev Disabil. 45 (2015) 14-20.

[24] Llorens-Martín M, Torres-Alemán I, Trejo JL. Pronounced individual variation in the response to the stimulatory action of exercise on immature hippocampal neurons. Hippocampus. 16 (2006) 480-490.

[25] He N, Jin WL, Lok KH, et al. Amyloid- $\beta$ (1-42) oligomer accelerates senescence in adult hippocampal neural stem/progenitor cells via formylpeptide receptor 2. Cell Death Dis. (2013) 2013;4:e924.

[26] Paz-Miguel JE, Flores R, Sánchez-Velasco P, et al.Reactive oxygen intermediates during programmed cell death induced in the thymus of the Ts(1716)65Dn mouse, a murine model for human Down's syndrome. J Immunol. 163 (1999) 5399-5410.

[27] Garlet TR, Parisotto EB, de Medeiros G, et al. Systemic oxidative stress in children and teenagers with Down syndrome. Life Sci. 93 (2013) 558-563.

[28] Butterfield DA, Di Domenico F, Swomley AM, Head E, Perluigi M. Redox proteomics analysis to decipher the neurobiology of Alzheimer-like neurodegeneration: overlaps in Down's syndrome and Alzheimer's disease brain. Biochem J. 463 (2014) 177-89.

[29] de Haan JB, Wolvetang EJ, Cristiano F, et al. Reactive oxygen species and their contribution to pathology in Down syndrome. Adv Pharmacol. 38 (1997) 379-402.

[30] Sinet PM, Michelson AM, Bazin A, et al. Increase in glutathione peroxidase activity in erythrocytes from trisomy 21 subjects. Biochem Biophys Res Commun. 67 (1975) 910-915.

[31] Hamed RR, Maharem TM, Abdel-Meguid N, et al. Purification and biochemical characterization of glutathione S-transferase from Down syndrome and normal children erythrocytes: a comparative study. Res Dev Disabil. 32 (2011) 1470-1482.

[32] Hayes JD, McLellan LI. Glutathione and glutathione-dependent enzymes represent a co-ordinately regulated defence against oxidative stress. Free Radic Res. 31 (1999) 273-300.

[33] Sebastià J, Cristòfol R, Pertusa M, et al. Down's syndrome astrocytes have greater antioxidant capacity than euploid astrocytes. Eur J Neurosci. 20 (2004) 2355-2366.

[34] Rodriguez C, Mayo JC, Sainz RM, et al. Regulation of antioxidant enzymes: a significant role for melatonin. J Pineal Res. 36 (2004) 1-9.

[35] Manchester LC, Coto-Montes A, Boga JA, Andersen LP, Zhou Z, Galano A, Vriend J, Tan DX, Reiter RJ. Melatonin: an ancient molecule that makes oxygen metabolically tolerable. J Pineal Res. 59 (2015) 403-19.

[36] Hardeland R. Antioxidative protection by melatonin: multiplicity of mechanisms from radical detoxification to radical avoidance.Endocrine. 27 (2005) 119-30.

[37] Olcese JM, Chao C, Mori T, et al. Protection against cognitive deficits and markers of neurodegeneration by long-term oral administration of melatonin in a transgenic model of Alzheimer disease. J Pineal Res. 47 (2009) 82-96.

[38] Feng Z, Qin C, Chang Y, et al. Early melatonin supplementation alleviates oxidative stress in a transgenic mouse model of Alzheimer's disease. Free Radic Biol Med. 40 (2006) 101-109.

[39] Lott IT. Antioxidants in Down syndrome.Biochim Biophys Acta. 1822 (2012) 657-663. 
[40] Tan X, Guo X, Liu H. Melatonin attenuates hippocampal neuron apoptosis and oxidative stress during chronic intermittent hypoxia via up-regulating B-cell lymphoma-2 and down-regulating B-cell lymphoma-2associated X protein. Saudi Med J. 34 (2013) 701-708.

[41] Jang YJ, Hong HN, Lee JD, Hwang O. Down-regulation of GTP cyclohydrolase I and tetrahydrobiopterin by melatonin. Neuroreport. 11 (2000) 3627-3630.

[42] Bekyarova G, Apostolova M, Kotzev I. Melatonin protection against burn-induced hepatic injury by down-regulation of nuclear factor kappa B activation. Int J Immunopathol Pharmacol. 25 (2012) 591-596.

[43] Proietti S, Cucina A, D'Anselmi F, Dinicola S, Pasqualato A, Lisi E, Bizzarri M. Melatonin and vitamin D3 synergistically down-regulate Akt and MDM2 leading to TGF $\beta$-1-dependent growth inhibition of breast cancer cells. J Pineal Res. 50 (2011) 150-158.

[44] Caballero B, Vega-Naredo I, Sierra V, Huidobro-Fernández C, Soria-Valles C, De Gonzalo-Calvo D, Tolivia D, Gutierrez-Cuesta J, Pallas M, Camins A, Rodríguez-Colunga MJ, Coto-Montes A. Favorable effects of a prolonged treatment with melatonin on the level of oxidative damage and neurodegeneration in senescence-accelerated mice. J Pineal Res. 45 (2008) 302-311.

[45] Tan DX, Manchester LC, Reiter RJ, Qi WB, Karbownik M, Calvo JR. Significance of melatonin in antioxidative defense system: reactions and products. Biol Signals Recept. 9 (2000) 137-59.

[46] Baydas G, Ozveren F, Akdemir I, Tuzcu M, Yasar A. Learning and memory deficits in rats induced by chronic thinner exposure are reversed by melatonin. J Pineal Res. 39 (2005) 50-56.

[47] Manda K, Anzai K, Kumari S, Bhatia AL. Melatonin attenuates radiation-induced learning deficit and brain oxidative stress in mice. Acta Neurobiol Exp. 67 (2007) 63-70.

[48] Rueda N, Florez J, Martinez-Cue C. Effects of chronic administration of SGS-111 during adulthood and during the pre- and post-natal periods on the cognitive deficits of Ts65Dn mice, a model of Down syndrome. Behav Brain Res. 188 (2008) 355-367.

[49] Ramirez-Rodriguez F, Klempin F, Babu H, et al. Melatonin modulates cell survival of new neurons in the hippocampus of adult mice. Neuropsychopharmacology. 34 (2009) 2180-2191.

[50] Manda K, Ueno M, Anzai K. Cranial irradiation-induced inhibition of neurogenesis in hippocampal dentate gyrus of adult mice: attenuation by melatonin pretreatment. J Pineal Res. 46 (2009) 71-78.

[51] Crupi R, Mazzon E, Marino A, et al. Melatonin's stimulatory effect on adult hippocampal neurogenesis in mice persists after ovariectomy. J Pineal Res. 51 (2011) 353-360. 


\section{Figure legends}

Fig. 1. Mean \pm S.E.M. of the levels of PC $(A)$ and TBARS $(B)$ in the cortex and hippocampus of TS and CO mice treated with melatonin or vehicle. ${ }^{*} p<0.05$, TS vs. CO; ${ }^{p}<0.05 ;{ }^{\#} p<0.01$; $\#$ \# $<0.001$, vehicle-treated vs. melatonin-treated mice. $p$ values reflect the outcomes of post hoc tests following a Bonferroni correction performed after MANOVAs.

Fig. 2. Mean \pm S.E.M. of the activity levels of different antioxidant enzymes in the hippocampus and cortex of TS and CO mice treated with melatonin or vehicle. Superoxide dismutase (SOD, A), catalase $(C A T, B)$, glutathione peroxidase $(G P x, C)$, glutathione reductase $(G R, D)$, and glutathione S-transferase (GST, E). Mean \pm SEM. ${ }^{*} p<0.05 ;{ }^{* *} p<0.01 ;{ }^{* *} p<0.001$ TS vs. CO. $p$ values reflect the outcomes of post hoc tests following a Bonferroni correction performed after MANOVAs.

Fig. 3. Representative photomicrographs of SA- $\beta$-gal-stained sections $(A)$ and mean \pm S.E.M. of the density of $\beta$-gal-positive cells (B) of the SGZ of the hippocampus of TS and CO mice treated with melatonin or vehicle. ${ }^{*} p<0.05$; TS vs. $C 0$; $\# p<0.05$; melatonin vs. vehicle. $p$ values reflect the outcomes of post hoc tests following a Bonferroni correction performed after MANOVAs.

Fig. 4. Schematic diagram summarizing the differences in the brain oxidative status between TS and $\mathrm{CO}$ mice and the effects produced by melatonin treatment in the cortex and hippocampus of TS mice. 
Fig. 1
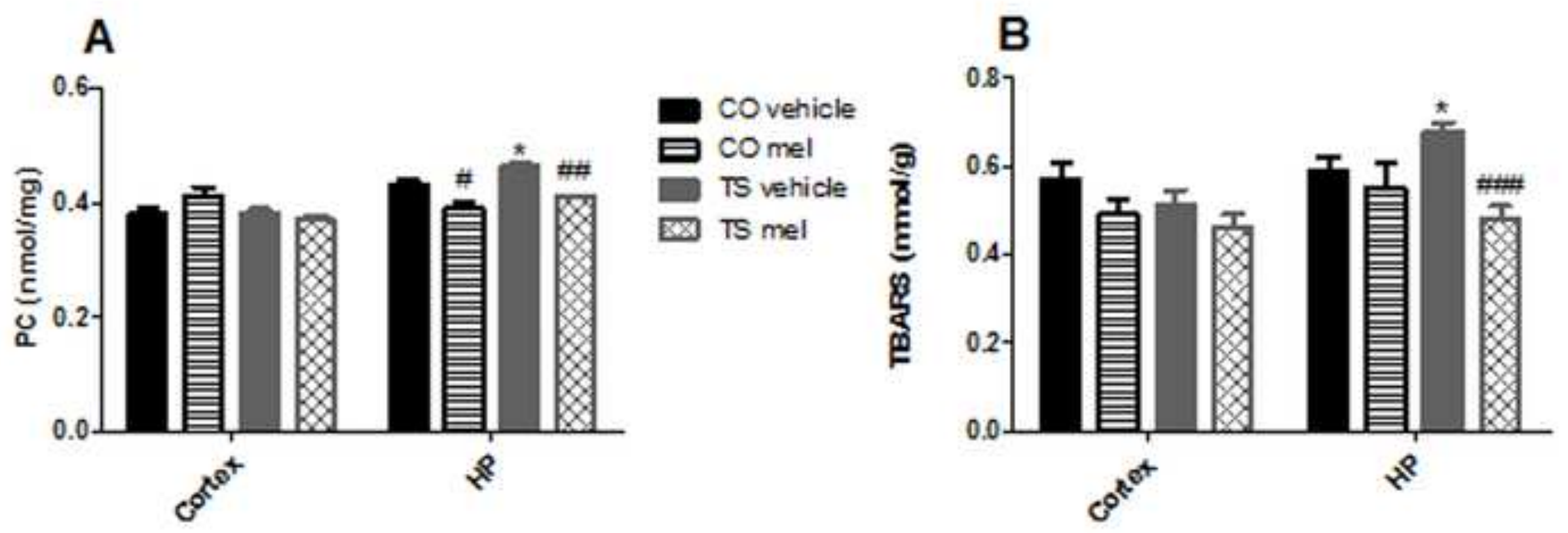
Fig. 2

A

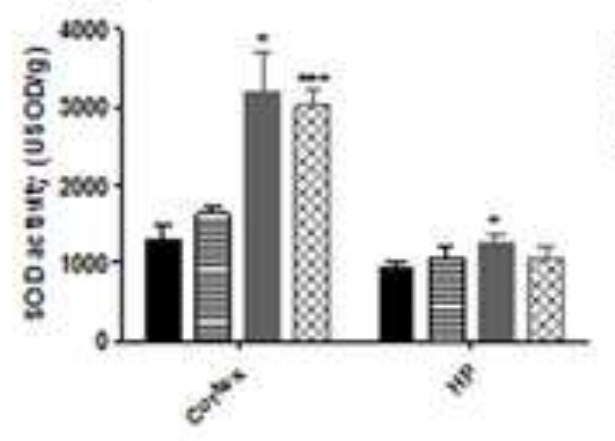

C

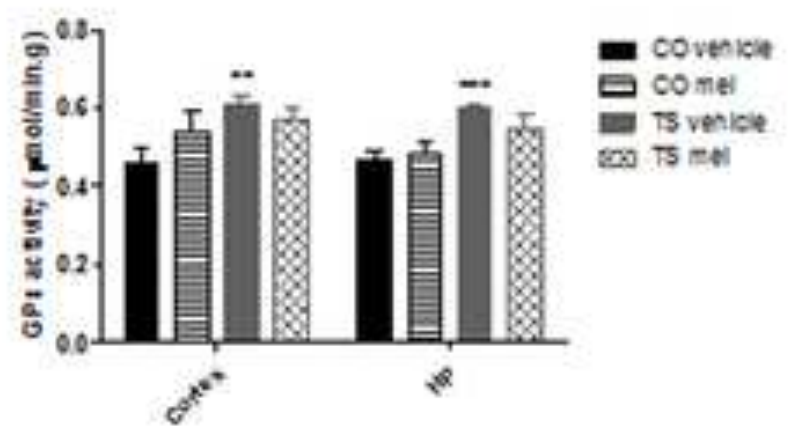

E

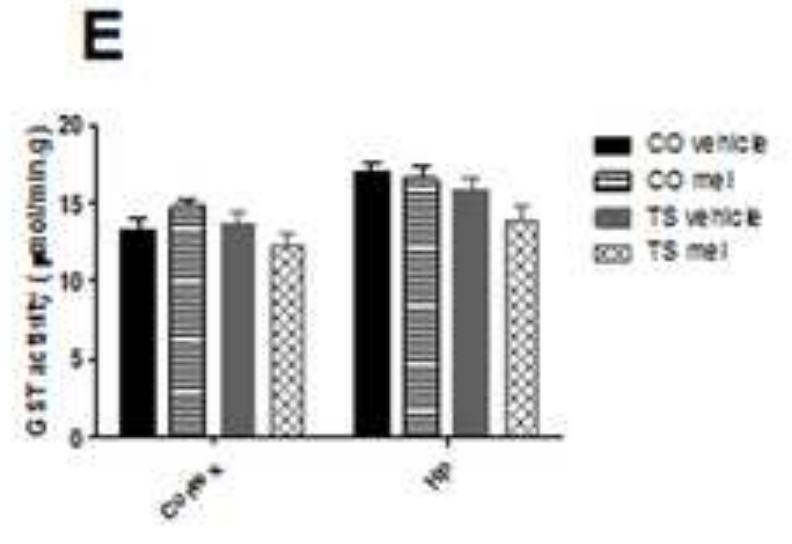

- covenice

日 $00 \mathrm{mat}$

D come

- TS venicle $\infty$ Ts mel
B

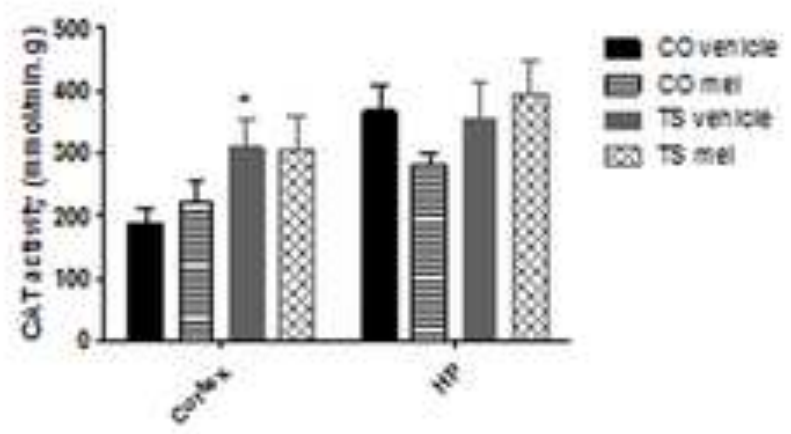

D

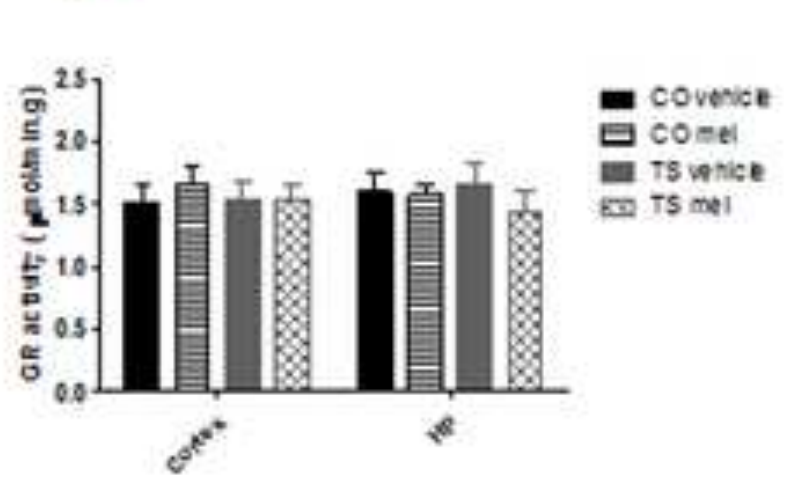


Fig. 3

A

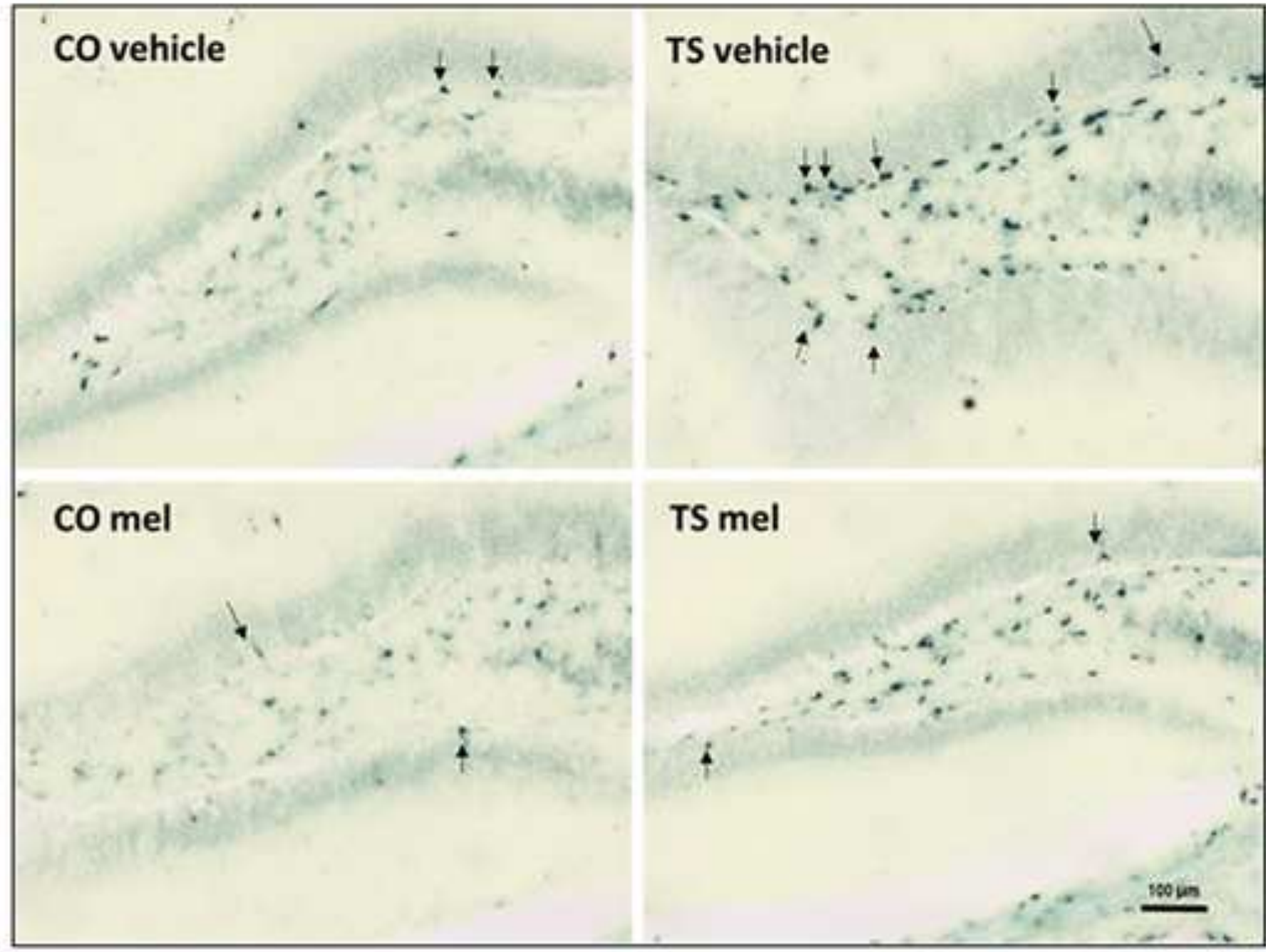

B

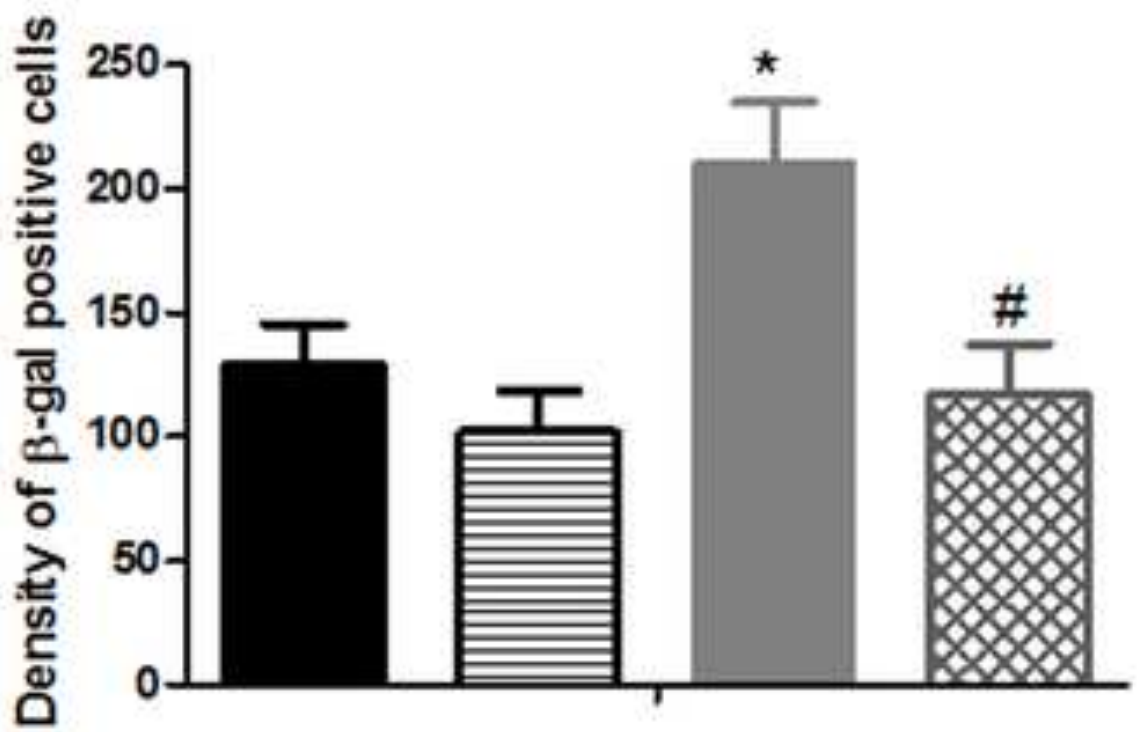

- CO vehicle

曰 $\mathrm{CO}$ mel

TS vehicle

四 TS mel 
Fig 4.

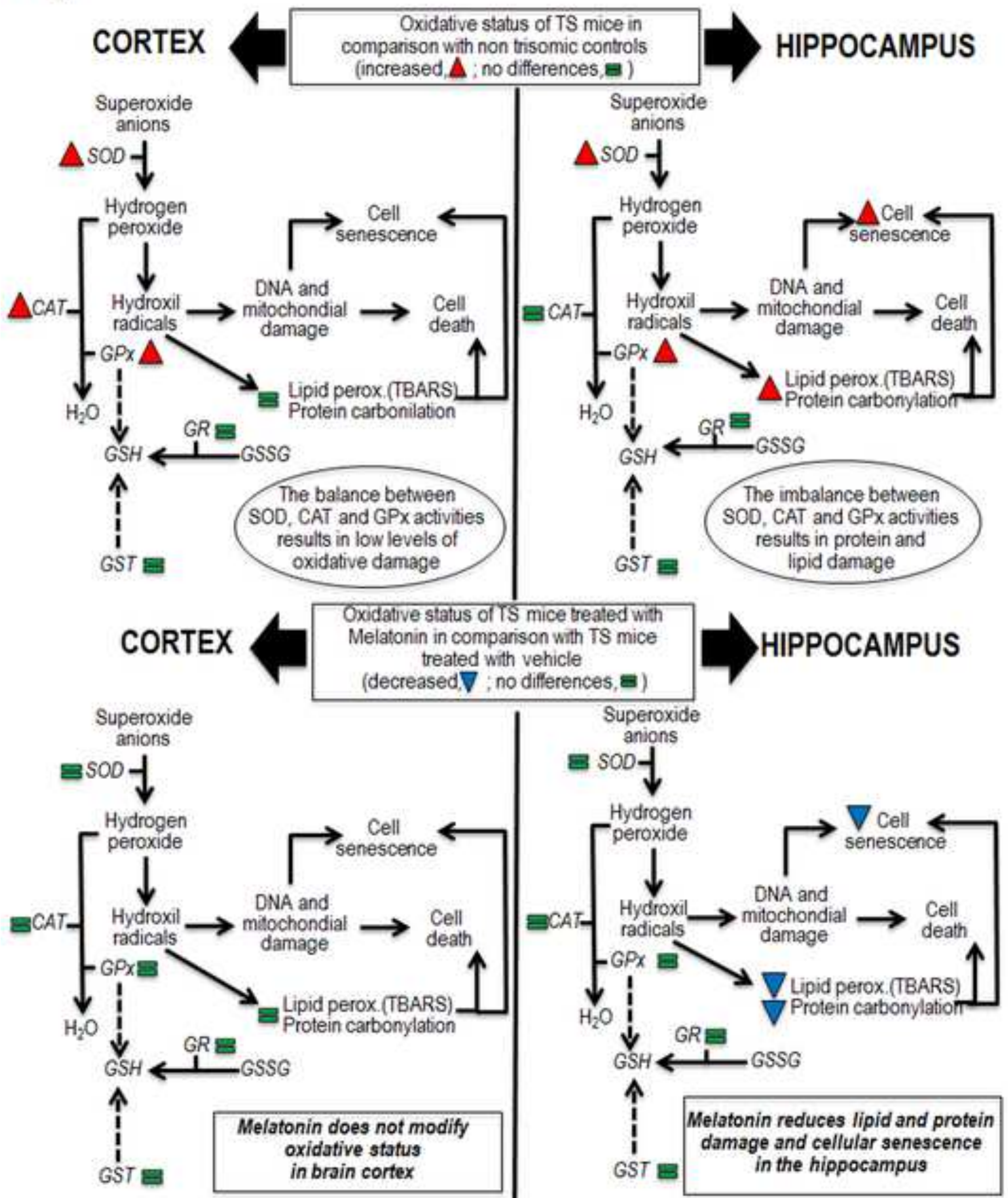

